\title{
Array Pattern Synthesis Using Particle Swarm Optimization with Dynamic Inertia Weight
}

\author{
Chuang Han and Ling Wang \\ School of Electronics and Information, Northwestern Polytechnical University, Xian 710072, China \\ Correspondence should be addressed to Chuang Han; hanchuang357@163.com
}

Received 22 January 2016; Accepted 16 March 2016

Academic Editor: Jaume Anguera

Copyright ( 2016 C. Han and L. Wang. This is an open access article distributed under the Creative Commons Attribution License, which permits unrestricted use, distribution, and reproduction in any medium, provided the original work is properly cited.

\begin{abstract}
A Feedback Particle Swarm Optimization (FPSO) with a family of fitness functions is proposed to minimize sidelobe level (SLL) and control null. In order to search in a large initial space and converge fast in local space to a refined solution, a FPSO with nonlinear inertia weight algorithm is developed, which is determined by a subtriplicate function with feedback taken from the fitness of the best previous position. The optimized objectives in the fitness function can obtain an accurate null level independently. The directly constrained SLL range reveals the capability to reduce SLL. Considering both element positions and complex weight coefficients, a low-level SLL, accurate null at specific directions, and constrained main beam are achieved. Numerical examples using a uniform linear array of isotropic elements are simulated, which demonstrate the effectiveness of the proposed array pattern synthesis approach.
\end{abstract}

\section{Introduction}

There are many synthesis methods for array pattern of minimum sidelobe level (SLL) and null control [1-3]. These proposed methods attempt to find the best solution for sensors position distribution or complex weight coefficients of linear array. Compared with equally spaced arrays, unequally spaced arrays with optimally spaced sensors have advantages including their capability of achieving higher spatial resolutions or lower sidelobe, or we can use fewer sensors to meet similar pattern specifications by carefully designing the locations of array sensors [4-6]. By changing the amplitudes and phases of the array elements' complex weights without any physical changes in the array, the method becomes suitable for adaptive processing applications in which the array pattern is dynamically adapted to the environments [7-10].

Although many studies have been published on array pattern synthesis, further research is still needed for the problems described below.

Firstly, only sensor positions are considered for minimum SLL and null control in [1,5]. In [5], one example discussed the geometry of a 28 -element array designed for SLL suppression in the region $\left[0^{\circ}, 180^{\circ}\right]$ and prescribed nulls at three groups of symmetric direction. It shows that a broad null $-50 \mathrm{~dB}$ deep is easily achieved by Particle Swarm Optimization (PSO). For the same condition, $\mathrm{PM}_{4} \mathrm{HPSO}-\mathrm{TVAC}$ achieves a performance for synthesizing unequally spaced linear array with low SLL and null control. However, the constrained SLL is not an accurate null level but lower than one default value by considering sensor positions.

Secondly, many techniques proposed in the literature adjust the amplitude and phase of the array element to achieve a low SLL, if desired, nulls at specific directions with constrained main beam [7-10]. Phase only control widely used in phased arrays to provide beam scanning is inexpensive to produce and, also, is more likely to minimize excitation errors and preserve coherence [8]. Only excitation amplitudes of each element are taken as optimization parameters using Taylor distribution and PSO in [9]. The single optimized parameter may limit the effectiveness of SLL. In [7], numerical examples are used to demonstrate the effectiveness of achieving an accurate null level by changing the complex weights. Three nulls of $-80 \mathrm{~dB}$ with a minimum average pattern value of 0.28 are achieved for an equally spaced linear array of 10 elements. However, the null level has a certain deviation with decreasing the number of elements even though the fitness 
has a large weight and the SLL has no obvious advantages by minimizing the pattern average level. It is likely to achieve the truly accurate null level and minimum SLL when both the weights and the positions are considered simultaneously with suitable fitness.

Thirdly, a simulated annealing (SA) based method was proposed in [11] to design an asymmetric array by optimizing both the sensor positions over a grid space and array complex weight coefficients. It does not simultaneously optimize all the parameters but perturbs the weight coefficient and position of each sensor in turn. It is possible that sparse arrays with continuously spaced sensors could have a high degree of freedom in lowering the SLL [4]. In [12], an evolutionary method based on backtracking search optimization algorithm (BSA) is proposed for linear antenna array pattern synthesis with prescribed nulls at interference directions. Pattern nulling is obtained by controlling the amplitude, position, and phase of the antenna array elements, but the null depth is not constrained with a given value. An accurate null level with minimum SLL will be obtained by both considering position and complex weights in this paper.

The challenge of determining optimum parameter values simultaneously stems from the nonlinear and nonconvex dependency of the array factor to the weights and the sensor positions [4]. In [13], the authors revealed that the synthesis of nonuniform array elements' positions, excitations, and phases is a complicated nonlinear problem which contains a number of decision variables. The performance of the employed optimization scheme is an important factor in the success of a pattern synthesis method, in terms of solution quality, computational load, and stability. PSO has received a lot of attention due to its simplicity of implementation and its capability to escape from the traps of local optima $[5,14]$.

A lot of research is being carried out to address the main limitation of PSO, which is its tendency to converge prematurely at local optima $[15,16]$. Modifications in the velocity calculation of PSO algorithm are proposed in [17]. The authors proposed that personal best influence and initial velocity values have an important influence on the search procedure. In [5], the authors revealed that one of the aspects of PSO's capability to find the global optima mainly depends upon its capability to explore the search space. The initial higher value of inertia weight applied to the last velocity improves the exploration of search space and its lower value towards the end of search helps to attain faster convergence [5].

Without loss of generality, the elements of the antenna array in this paper are isotropic radiators. However, the method can be generalized for different types of antennas, such as microstrip antenna and horn antenna. In [18], the authors presented a novel patch antenna design with high directivity in the broadside direction by using genetic algorithms (GA). This type of antenna has more advantage for the optimization of array pattern synthesis. Implementing the proposed method to other types of antennas is investigating by the authors. Progress will be shown in a separate paper.

In this paper, both element positions and complex weight coefficients are optimized for minimum SLL and accurate null level at specific directions with constrained main beam.
Numerical examples are used to demonstrate the effectiveness of the proposed array pattern synthesis approach and the novelty of this paper will be described as follows:

(i) The optimized objectives in the fitness function can obtain an accurate null level. The directly constrained SLL range reveals the capability to reduce SLL. In [7], the null level has no an accurate level, even though there is a big weighting factor for null control in the fitness. The objective of minimizing the pattern average value has influence on the null control and SLL minimization.

(ii) Considering both element positions and complex weight coefficients, a low-level SLL, accurate null at specific directions, and constrained main beam are achieved.

(iii) Feedback Particle Swarm Optimization (FPSO) is proposed for a large initial search space and fast convergence in local space with refined solution.

The remaining part of this paper is organized as follows. In Section 2, the array synthesis formulations for unequally linear array and PSO with initial conditions are briefly discussed. The FPSO method and different fitness functions are proposed and discussed in Section 3. Section 4 describes numerical examples and shows the comparative performance of the presented technique. Concluding remarks are given in Section 5.

\section{Method on Array Synthesis Using PSO}

Consider a uniformly planar array of $N$ isotropic elements with a spacing of $d_{i}$ as shown in Figure 1 . The position of each sensor along the $x$-axis can be written as $x_{n}$. We just consider the array factor, which can be expressed as

$$
F(\theta)=\sum_{n=1}^{N} w_{n} \exp \left(\frac{j 2 \pi x_{n} \sin \theta}{\lambda}\right),
$$

where

$$
x_{n}= \begin{cases}\sum_{i=1}^{n-1} d_{i}, & \text { for } n \geq 2 \\ 0, & \text { for } n=1\end{cases}
$$

and $\theta$ (measured from $z$-axis) is the pitch angle of radiation for the transmit array and the incidence of the plane wave for the receive array. $\lambda$ is the wavelength and $w_{n}$ is the weight coefficient of the $n$th sensor. Since $w_{n}$ is complex, it can be expressed as $w_{n}=\beta_{n} \exp \left(j \phi_{n}\right)$ where $\beta_{n}$ and $\phi_{n}$ are the amplitude and phase of $w_{n}$, respectively. Consequently, the array factor can be expressed as

$$
F(\theta)=\sum_{n=1}^{N} \beta_{n} \exp \left[j\left(\frac{2 \pi \sum_{i=2}^{n} d_{i} \sin \theta}{\lambda}+\phi_{n}\right)\right] .
$$

Particle Swarm Optimization (PSO), also known as swarm intelligence, is a robust stochastic evolutionary computation technique based on the movement of intelligent 


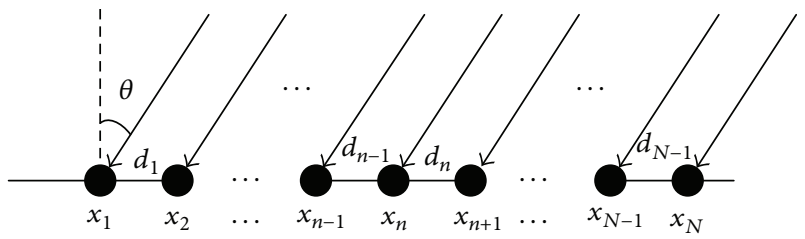

Figure 1: Geometry of the $N$-elements' unequally linear array placed along the $x$-axis.

swarms. The position and the velocity relationship after the $t$ th iteration between any two individuals are obtained by the following updating formula:

$$
\begin{aligned}
\mathbf{v}_{i, t+1}= & \mu_{t} \mathbf{v}_{i, t}+\tau_{1} \operatorname{rand}_{i, 1}\left(\mathbf{p}_{i, t}-\boldsymbol{\alpha}_{i, t}\right) \\
& +\tau_{2} \operatorname{rand}_{i, 2}\left(\mathbf{g}_{t}-\boldsymbol{\alpha}_{i, t}\right), \\
\boldsymbol{\alpha}_{i, t+1}= & \boldsymbol{\alpha}_{i, t}+\mathbf{v}_{i, t+1},
\end{aligned}
$$

where $\mu_{t}$ is a parameter called the inertia weight and the acceleration factors $\tau_{1}$ and $\tau_{2}$ are positive constants that control the relative impact of the personal (local) and common (global) knowledge on the movement of each particle with values ranging from 1.5 to 2.05 . The terms $\operatorname{rand}_{i, 1}$ and $\operatorname{rand}_{i, 2}$ are independent, uniformly distributed random variables in the range of 0 to 1 , and $\mathbf{p}_{i, t}$ is the best previous position of $\boldsymbol{\alpha}_{i, t}$ while $\mathbf{g}_{t}$ is the best overall position achieved by a particle within the entire population.

The initial swarm is formed by using a uniform array with an intersensor spacing of $\lambda / 2$ for each sensor and the complex weight coefficients of steering vectors where $w_{n}=$ $e^{-j 2 \pi x_{n} \sin \theta_{S} / \lambda}$. Besides the design of the array pattern with element positions and complex weight coefficients discussed in [4], an extensive study has been made on the performance of the PSO under different conditions in the following sections, where several configurations and weights constraints were considered. The performance of these designs will be compared with those reported in [7] in terms of the null level, the SLL, and the convergence speed. Without loss of generality, we set the parameters of (3) to

$$
\begin{aligned}
0 & \leq \beta_{n} \leq 2 \quad i=1,2, \ldots, N, \\
-\pi & \leq \phi_{n} \leq \pi \quad i=1,2, \ldots, N, \\
0.25 \lambda & \leq d_{i} \leq 0.75 \lambda \quad d_{1}=0, i=2,3, \ldots, N .
\end{aligned}
$$

To have the same range of complex weights used in [4,7], we set the amplitude between 0 and 2 and the phase between $-\pi$ and $\pi$ for every sensor. Here, we adopt the constraints of intersensor spacing between $0.25 \lambda$ and $0.75 \lambda$ which has a smaller search range than that of [5].

\section{FPSO and Different Fitness}

In [5], it was noted that the main limitation of PSO is its tendency to converge prematurely at local optima, and in [4], the iteration numbers to converge or the number of the fitness function evaluations was identified as an investigative topic.
In this paper, the solution includes amplitudes and phases constrained independently or with element positions simultaneously. A case study reveals that the choice of mutation probability and mutation step size has a strong influence on the convergence behavior of the swarm. For more mutation probability, there should be a large random velocity or large weighting coefficient for the velocity update before entering the global optimization range. To enhance the search of the precise solution that affects the index of pattern, a small value of velocity and the weighting coefficient are important for the absolute best value.

The concept of linearly decreasing inertia weight applied to Particle Swarm Optimization (LPSO) and the method of parameter strategy for PSO were proposed using the overshoot and the peak time of a transition in [15]. In [19], dynamic inertia weight PSO (DIW-PSO) was proposed where the inertia weight for every particle is dynamically updated based on the feedback taken from the fitness of the best previous position found by the particle.

For a large initial search space and fast convergence in local space with refined solution, we propose a nonlinear inertia weight decided by subtriplicate function with feedback taken from the fitness of the best previous position. The feedback function with the inertia weight can be expressed as

$$
\mu_{t}=\frac{\mu_{\max }+\mu_{\min }}{2}+\frac{\mu_{\max }-\mu_{\min }}{2} \gamma \sqrt[3]{F_{\mathrm{FB}}-F_{\mathrm{OP}}-\kappa},
$$

where $\mu_{t}$ is limited between $\mu_{\max }$ and $\mu_{\min }$ which are the initial and final values of the inertia weight and set to 0.7 and 0.4 , respectively. The terms $\gamma$ and $\kappa$ are independent to control the slope and location of the feedback function. $F_{\mathrm{OP}}$ is the expected fitness value of optimal solution and is set to zero for this paper. $F_{\mathrm{FB}}$ is the feedback fitness value taken from the best previous position found by the particle.

For different optimization accuracy or kinds of array pattern synthesis and different steering angles, we can set $\gamma$ and $\kappa$ to appropriate values. $F_{\mathrm{OP}}$ will be a prior value through several experiments. In the next section, we describe the performance for different optimal conditions with these sets of parameters.

As the objective of optimization is to minimize the SLL and null control of the array pattern by adjusting the parameters of the array, subject to the given design specifications and constraints, the fitness function can be defined as

$$
\text { fit } 1=a \sum_{i=1}^{J}|| \frac{P\left(\theta_{i}\right)}{P\left(\theta_{S}\right)}\left|-10^{N_{i} / 20}\right|+\left|\frac{P_{\mathrm{av}}(\theta)}{P\left(\theta_{S}\right)}\right|
$$

which is the same as the fitness of [7], and where $N_{i}$ is the $i$ th normalized pattern null value prespecified in $\mathrm{dB}$ corresponding to the $i$ th jammer, $\theta_{i}$ is the direction of $i$ th jammer, $\theta_{S}$ is the direction of signal, $a$ is the weighting factor used to increase the value of the fitness function's first term, and $P_{\mathrm{av}}$ is the pattern average value with $N_{P}$ points at which the pattern values are calculated, and it could be computed as follows:

$$
P_{\mathrm{av}}(\theta)=\frac{\sum_{\theta}|P(\theta)|}{N_{P}} .
$$


In [7], $a$ may have a large value of 100, and the first term of fitness has an important role in the optimal process. In practical examples, the value of $\left|P\left(\theta_{i}\right)\right|$ may not be the accurate null level of $N_{i}$, because the value of $\left|P\left(\theta_{S}\right)\right|$ may miss the maximum value of pattern. Thus, we divide the fitness function into three objectives as shown in (10). Further, for getting a truly minimum SLL, the estimate of SLL is set to an independent term shown in (11):

$$
\begin{aligned}
& \text { fit } 2=b_{1} \sum_{i=1}^{J} \| \frac{P\left(\theta_{i}\right)}{\max P(\theta)}\left|-10^{N_{i} / 20}\right| \\
& +b_{2}|| \frac{P\left(\theta_{S}\right)}{\max P(\theta)}|-1|+b_{3}\left|\frac{P_{\mathrm{av}}(\theta)}{\max P(\theta)}\right|, \\
& \text { fit } 3=c_{1} \sum_{i=1}^{J} \| \frac{P\left(\theta_{i}\right)}{\max P(\theta)}\left|-10^{N_{i} / 20}\right| \\
& +c_{2}|| \frac{P\left(\theta_{S}\right)}{\max P(\theta)}|-1| \\
& +c_{3}\left\{\max _{\theta_{\mathrm{SL}} \in\left[-90^{\circ}, \theta_{b}\right) \cap\left(\theta_{e}, 90^{\circ}\right]}\left|\frac{P\left(\theta_{\mathrm{SL}}\right)}{\max P(\theta)}\right|\right\} \text {. }
\end{aligned}
$$

The values of $b_{1}, b_{2}, b_{3}, c_{1}, c_{2}$, and $c_{3}$ are the weights for imposing penalty, $\theta_{\mathrm{SL}}$ is the spanned angles within the sidelobe band, and $\left[\theta_{b}, \theta_{e}\right]$ is the range of the mainlobe. For the fitness of (10) and (11), the objectives of the FPSO are

(i) minimizing the pattern SLL,

(ii) controlling the nulls to an accurate value,

(iii) maximizing the pattern value in the direction of the signal.

It is well known that array optimization should be organized along a specific trade-off rule between the sidelobe level and mainlobe width. To simplify this problem, only the above three objectives are considered in the optimization. The mainlobe width is fixed to be within a given range according to the design specifications in this work.

\section{Numerical Examples and Results Analysis}

To illustrate the effectiveness of the proposed method, we compare the performance of FPSO and different fitness with that of the GA [7]. To get a fair comparison, we use the same simulation setup of $N=10$, three jammers in the directions of $\theta_{i}=-45^{\circ}, 0^{\circ}, 10^{\circ}$ and one signal direction of $\theta_{S}=30^{\circ}$ in [7]. The range of sidelobe band is $\theta_{b}=10^{\circ}, \theta_{e}=50^{\circ}$, and the sampled point is $N_{P}=1800$ for $\theta$ which scans from $-90^{\circ}$ to $90^{\circ}$.

For the optimization of linear array, we search the best element positions and optimal complex weights to synthesize the pattern by FPSO in the following examples. The population size is set to 100 and the maximum number of allowable iterations is 200 . The computational complexity is measured by the product of the number of fitness functions evaluated and the number of parameters optimized, which

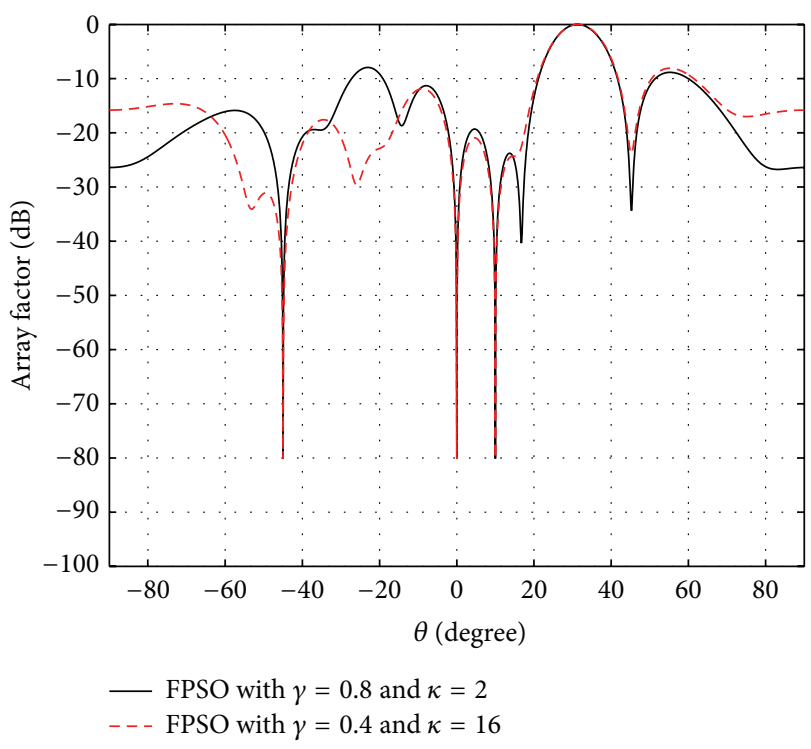

FIGURE 2: Resultant beam pattern designed $(N=10)$ by the feedback function of (8) with $\gamma=0.8, \kappa=2$ and $\gamma=0.4, \kappa=16$.

is a common way of estimating the computational complexity of evolutionary algorithms. In [7], uniform random initialization is used and the population size is selected to be ten times the number of the antenna array elements taking into consideration that two chromosomes will be used to represent each array element complex weight, one for the real part and the other for the imaginary part. In this method, the computational complexity is 100,000 with the maximum number of 500 generations.

Case 1 (linear array with optimum complex weights and equally spaced positions by Fit1). In this case, only complex weights are considered to optimize the pattern by fitness of (8) with $a=100$ which is the same with that of [7]. Figure 2 shows two beam patterns by feedback function (8) with $\gamma=0.8$, $\kappa=2$ and $\gamma=0.4, \kappa=16$. Table 1 shows the optimized results that the null levels are $-80.12 \mathrm{~dB}$ and $-80.14 \mathrm{~dB}$ with the maximum pattern point of $31^{\circ}$. The null level has not obtained an accurate value for the effect of $\left|P\left(\theta_{S}\right)\right|$ losing the maximum pattern.

In the two examples, the average pattern values are equal to 0.21 which is better than that of [7], and the SLL is $-7.9 \mathrm{~dB}$ and $-8.1 \mathrm{~dB}$ which is similar to that of [7]. Figure 3 shows the mean convergence of fitness values with iteration, which declined rapidly in the initial iteration and converged rapidly with a refined solution. The feedback function with $\gamma=0.4$, $\kappa=16$ has a faster speed of convergence than the feedback function with $\gamma=0.8, \kappa=2$. For the case of linear array with optimum complex weights and equally spaced positions, the computational complexity is 400,000 because of optimizing the two parameters of the amplitude and the phase.

Case 2 (optimum complex weights and equally spaced positions by different fitness). In this case, two fitness functions are used for FPSO to find the optimum complex weights. For an accurate null level relative to case 1 , the fitness function of 
TABLE 1: Normalized pattern average value, normalised pattern null values, and SLL for different solutions.

\begin{tabular}{lcccccc}
\hline Method & \multicolumn{3}{c}{ Null value (dB) } & Pattern average value & SLL (dB) & Computational complexity \\
& $\theta_{i}=-45^{\circ}$ & $\theta_{i}=0^{\circ}$ & $\theta_{i}=10^{\circ}$ & 0.28 & -8.0 & 1000,000 \\
[7] & -80.02 & -80.02 & -80.03 & 0.21 & -7.9 & 400,000 \\
Figure 2 solid & -80.12 & -80.12 & -80.12 & 0.21 & -8.1 & 400,000 \\
Figure 2 dashed & -80.14 & -80.14 & -80.14 & 0.24 & -9.1 & 400,000 \\
Figure 4 & -80.00 & -80.00 & -80.00 & 0.23 & -9.5 & 400,000 \\
Figure 5 & -80.00 & -80.00 & -80.00 & 0.19 & -14.0 & 600,000 \\
Figure 6 & -80.00 & -80.00 & -80.00 & & \\
\hline
\end{tabular}

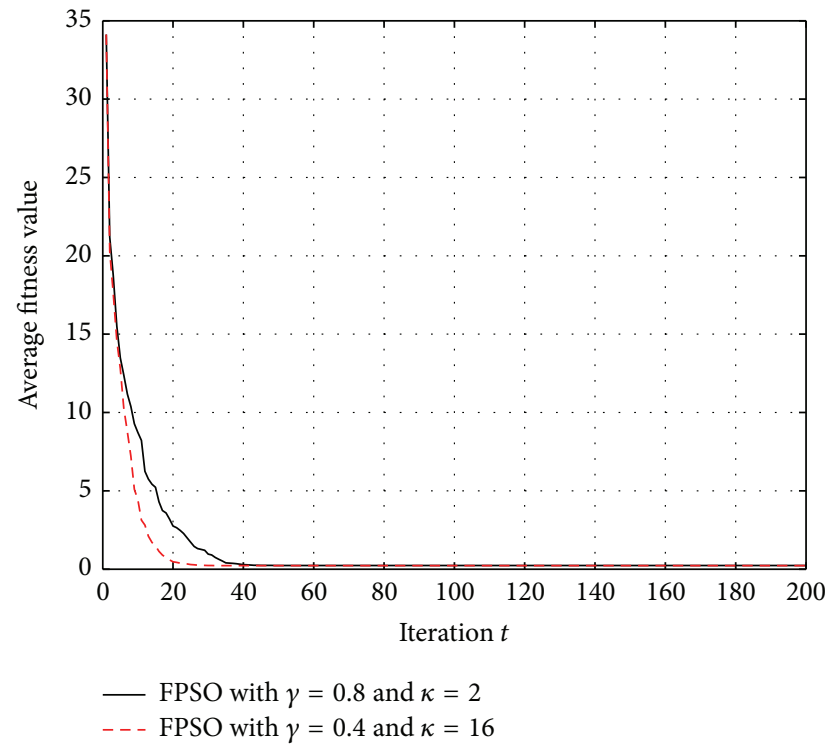

FIGURE 3: The mean convergence of fitness values with iteration (averaged over 10 trials) by the feedback function of (8) with $\gamma=0.8$, $\kappa=2$ and $\gamma=0.4, \kappa=16$.

(10) with $b_{1}=100, b_{2}=1, b_{3}=1$ is proposed to simulate the multiple objectives separately, and the result is shown in Figure 4 . The null level can get $-80.00 \mathrm{~dB}$ with the average pattern value of 0.24 . For (10), using FPSO, we can get a more accurate null level and 0.04 lower $P_{\text {av }}$ than that of [7].

In [7], it was noted that the term $\left|P_{\mathrm{av}} / P_{S}\right|$ could be considered as the normalized pattern average and, hence, it could be used as a measure of the SLL. However, we propose the fitness function of (11) which is more likely to achieve the truly lower SLL. We set $c_{1}=100, c_{2}=1, c_{3}=1$ for the fitness function of (11) optimized by FPSO. Figure 5 shows the result of the null level equal to $-80 \mathrm{~dB}$, a $P_{\mathrm{av}}$ value of 0.23 , and an SLL of $-9.5 \mathrm{~dB}$, which is $0.4 \mathrm{~dB}$ lower than that of the fitness function of (10).

Case 3 (optimum complex weights and unequally spaced positions). In this case, both element positions and complex weight coefficients are optimized for a minimum SLL and accurate null level at specific directions with a constrained main beam. The fitness function of (11) is used for FPSO, and the result is given in Figure 6. The null level has an

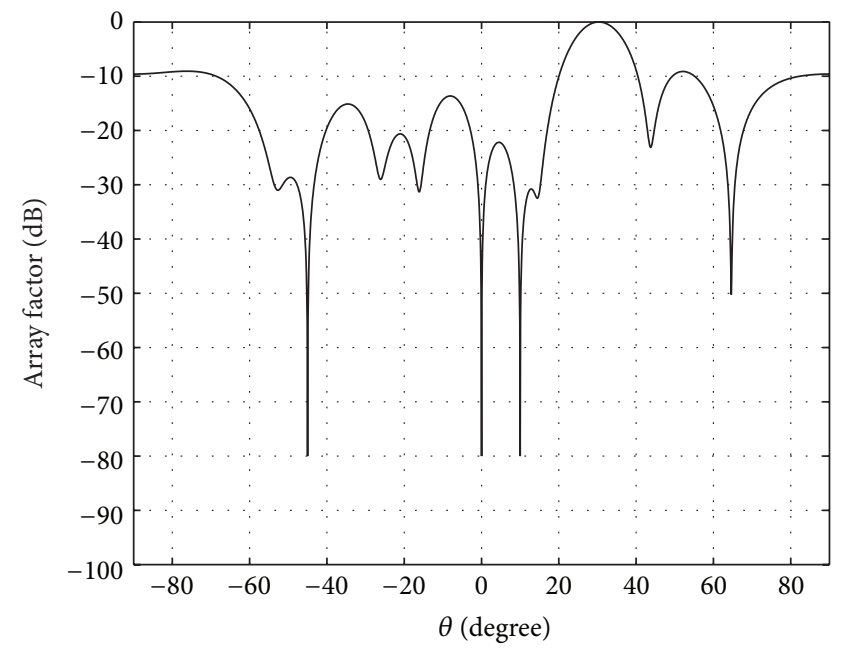

FIGURE 4: Resultant beam pattern designed $(N=10)$ by the feedback function of (10).

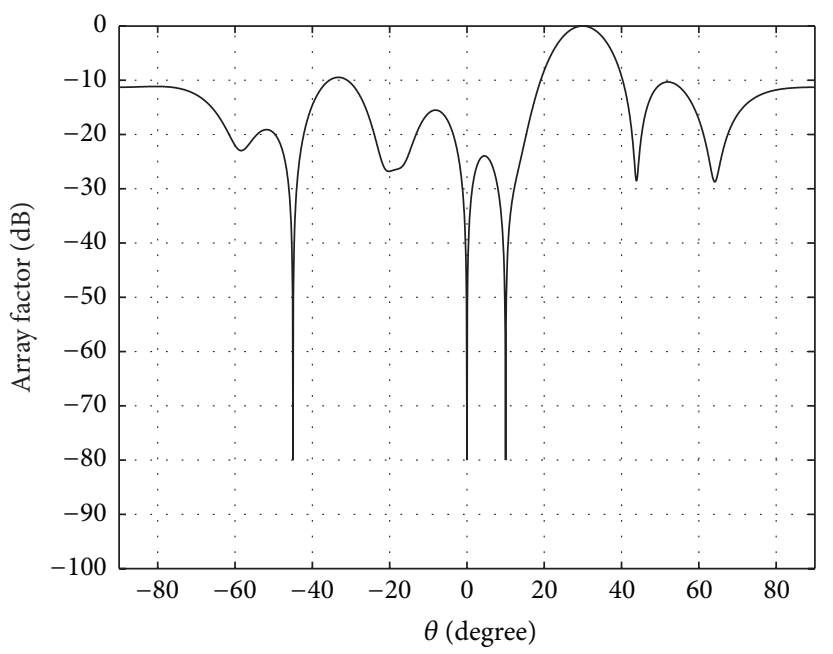

FIGURE 5: Resultant beam pattern designed $(N=10)$ by the feedback function of (11).

accurate value of $-80 \mathrm{~dB}$ and a $P_{\mathrm{av}}$ value of 0.19 . The obvious performance of this method is the SLL of $-14.0 \mathrm{~dB}$, which is $4.5 \mathrm{~dB}$ lower than that of only complex weights considered. For the case of optimum complex weights and unequally spaced positions, the computational complexity is 600,000 


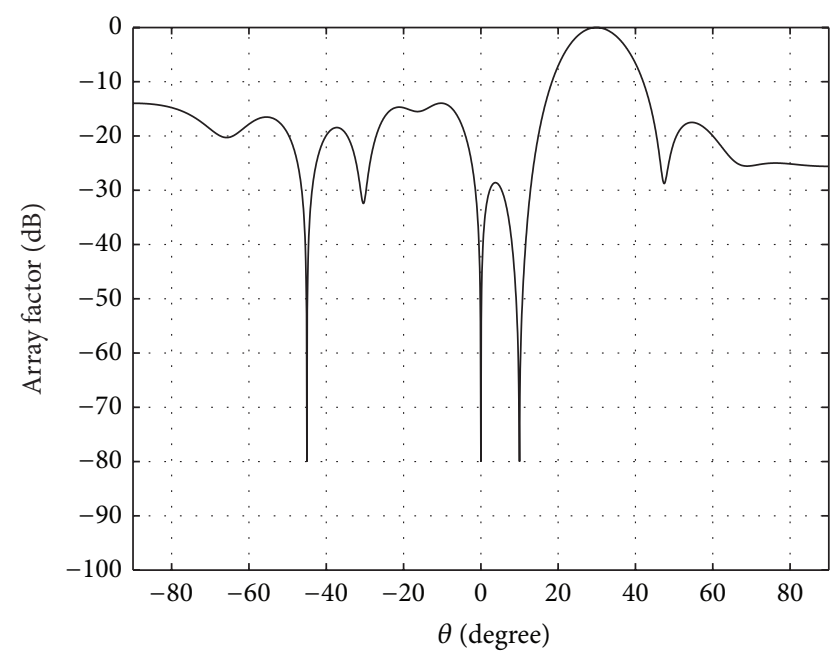

Figure 6: Resultant beam pattern designed $(N=10)$ by the feedback function of (11) considering both element positions and complex.

because of the extra parameter of position optimized. Even so, computational complexity is less than that of the method in [7].

\section{Conclusion}

The objective of the proposed method is to minimize the SLL and null control with a desired mainlobe by FPSO. Numerical examples show that the FPSO is able to achieve a lower average pattern value for the same fitness with [7]. FPSO has a rapid declined speed in the initial iteration and converges fast in local space to a refined solution with the appropriate parameters in the feedback function. The respectively optimized objectives in the fitness can obtain an accurate null level for its independence and the directly constrained SLL range has a better effect on the result of lower SLL. In this paper, the truly lower SLL and accurate null control have been realized by considering both element positions and complex weight coefficients.

\section{Competing Interests}

The authors declare that they have no competing interests.

\section{Acknowledgments}

This work was supported in part by the National Natural Science Foundation of China (nos. 61271416 and 61301093), the Fundamental Research Funds for the Central Universities (no. 3102014KYJD027), and NPU Foundation for Fundamental Research (no. JCY20130132).

\section{References}

[1] X. Wang, Y. Zhou, and Y. Wang, "An improved antenna array pattern synthesis method using fast fourier transforms," International Journal of Antennas and Propagation, vol. 2015, Article ID 316962, 9 pages, 2015.
[2] M. M. Khodier and C. G. Christodoulou, "Linear array geometry synthesis with minimum sidelobe level and null control using particle swarm optimization," IEEE Transactions on Antennas and Propagation, vol. 53, no. 8, pp. 2674-2679, 2005.

[3] K. Yang, Z. Zhao, and Q. H. Liu, "Fast pencil beam pattern synthesis of large unequally spaced antenna arrays," Institute of Electrical and Electronics Engineers. Transactions on Antennas and Propagation, vol. 61, no. 2, pp. 627-634, 2013.

[4] L. Cen, Z. L. Yu, W. Ser, and W. Cen, "Linear aperiodic array synthesis using an improved genetic algorithm," Institute of Electrical and Electronics Engineers. Transactions on Antennas and Propagation, vol. 60, no. 2, part 2, pp. 895-902, 2012.

[5] R. Bhattacharya, T. K. Bhattacharyya, and R. Garg, "Position mutated hierarchical particle swarm optimization and its application in synthesis of unequally spaced antenna arrays," IEEE Transactions on Antennas and Propagation, vol. 60, no. 7, pp. 3174-3181, 2012.

[6] X. R. Wang, E. Aboutanios, and M. G. Amin, "Thinned array beampattern synthesis by iterative soft-thresholding-based optimization algorithms," IEEE Transactions on Antennas and Propagation, vol. 62, no. 12, pp. 6102-6113, 2014.

[7] H. M. Elkamchouchi and M. M. Hassan, "Array pattern synthesis approach using a genetic algorithm," IET Microwaves, Antennas and Propagation, vol. 8, no. 14, pp. 1236-1240, 2014.

[8] T. H. Ismail and Z. M. Hamici, "Array pattern synthesis using digital phase control by quantized particle swarm optimization," IEEE Transactions on Antennas and Propagation, vol. 58, no. 6, pp. 2142-2145, 2010.

[9] S. Chatterjee, S. Chatterjee, and D. R. Poddar, "Synthesis of linear array using Taylor distribution and Particle Swarm Optimisation," International Journal of Electronics, vol. 102, no. 3, pp. 514-528, 2015.

[10] D. I. Abu-Al-Nadi, T. H. Ismail, H. Al-Tous, and M. J. Mismar, "Design of linear phased array for interference suppression using array polynomial method and particle swarm optimization," Wireless Personal Communications, vol. 63, no. 2, pp. 501513, 2012.

[11] A. Trucco, "Synthesizing asymmetric beam patterns," IEEE Journal of Oceanic Engineering, vol. 25, no. 3, pp. 347-350, 2000.

[12] K. Guney and A. Durmus, "Pattern nulling of linear antenna arrays using backtracking search optimization algorithm," International Journal of Antennas and Propagation, vol. 2015, Article ID 713080, 10 pages, 2015.

[13] M.-Y. Zheng, K.-S. Chen, H.-G. Wu, and X.-P. Liu, "Sparse planar array synthesis using matrix enhancement and matrix pencil," International Journal of Antennas and Propagation, vol. 2013, Article ID 147097, 7 pages, 2013.

[14] B. Sun, C. Liu, Y. Liu, X. Wu, Y. Li, and X. Wang, "Conformal array pattern synthesis and activated elements selection strategy based on PSOGSA algorithm," International Journal of Antennas and Propagation, vol. 2015, Article ID 858357, 7 pages, 2015.

[15] W. Zhang, D. Ma, J.-J. Wei, and H.-F. Liang, "A parameter selection strategy for particle swarm optimization based on particle positions," Expert Systems with Applications, vol. 41, no. 7, pp. 3576-3584, 2014.

[16] R. Bera, D. Mandal, R. Kar, and S. P. Ghoshal, "Application of particle swarm optimization technique in hexagonal and concentric hexagonal antenna array for side lobe level reduction," Advances in Intelligent Systems and Computing, vol. 343, pp. 333347, 2015. 
[17] A. Modiri and K. Kiasaleh, "Modification of real-number and binary PSO algorithms for accelerated convergence," IEEE Transactions on Antennas and Propagation, vol. 59, no. 1, pp. 214-224, 2011.

[18] J. Jayasinghe, J. Anguera, and D. Uduwawala, "Genetic algorithm optimization of a high-directivity microstrip patch antenna having a rectangular profile," Radioengineering, vol. 22, no. 3, pp. 700-707, 2013.

[19] A. Alfi, "Particle swarm optimization algorithm with dynamic inertia weight for online parameter identification applied to Lorenz chaotic system," International Journal of Innovative Computing, Information and Control, vol. 8, no. 2, pp. 1191-1203, 2012. 


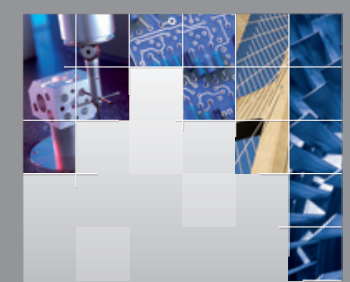

\section{Enfincering}
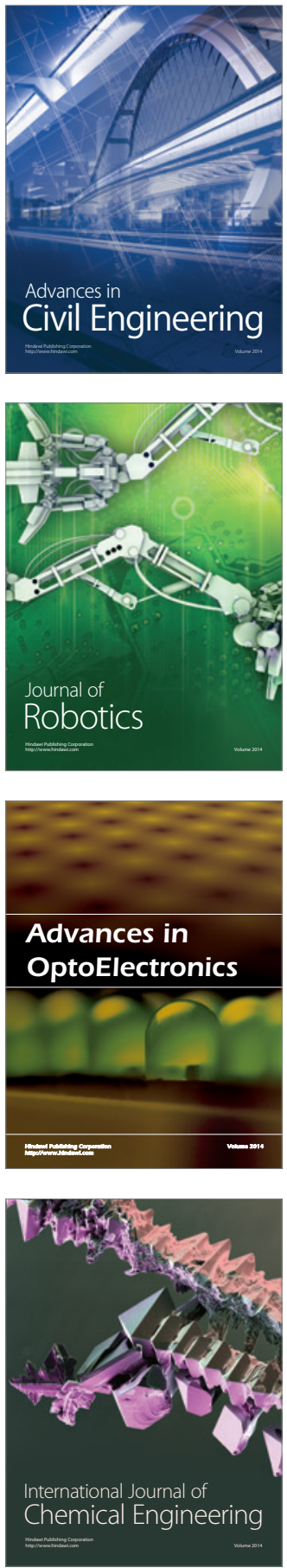

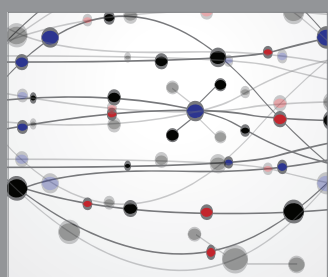

The Scientific World Journal

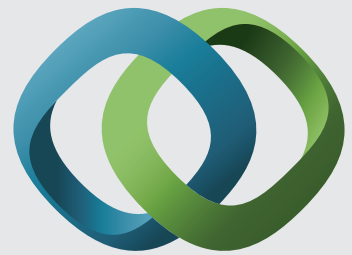

\section{Hindawi}

Submit your manuscripts at

http://www.hindawi.com
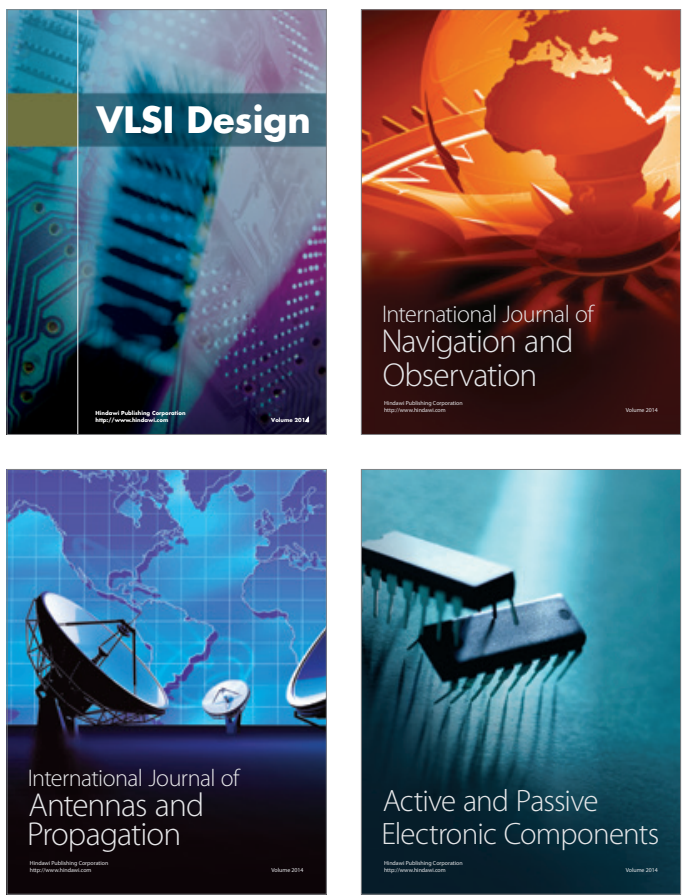
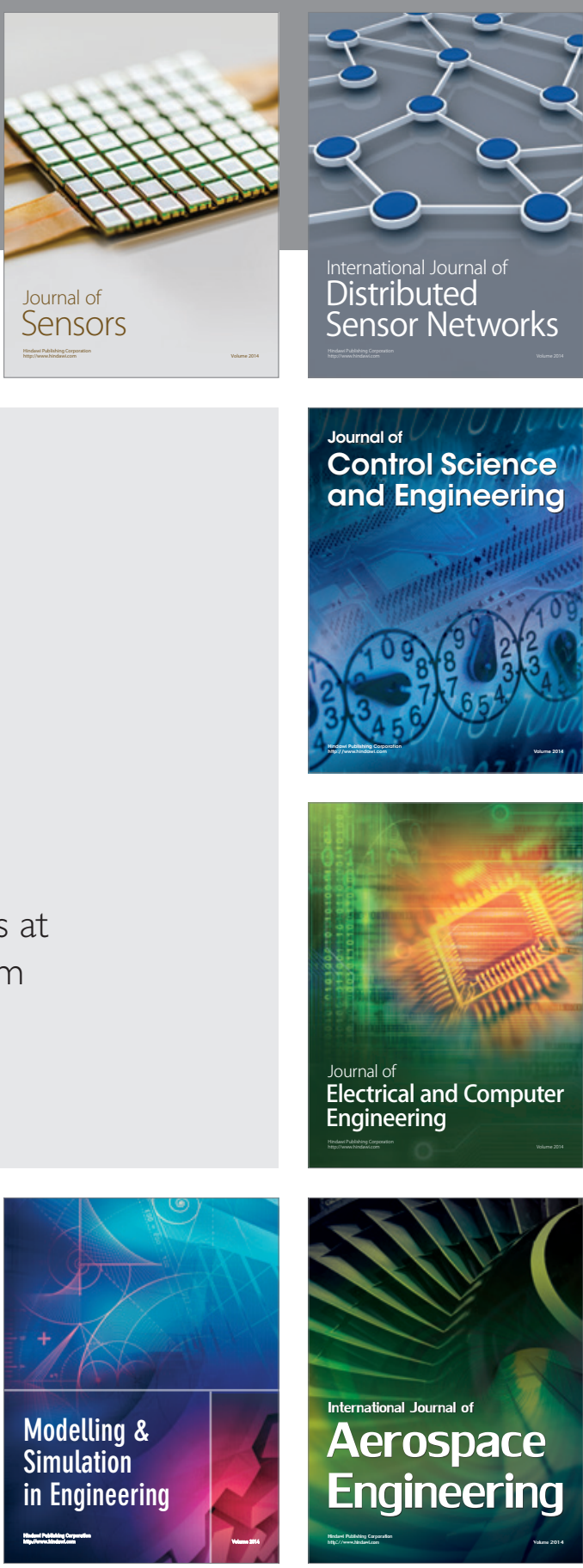

International Journal of

Distributed

Sensor Networks

Journal of

Control Science

and Engineering
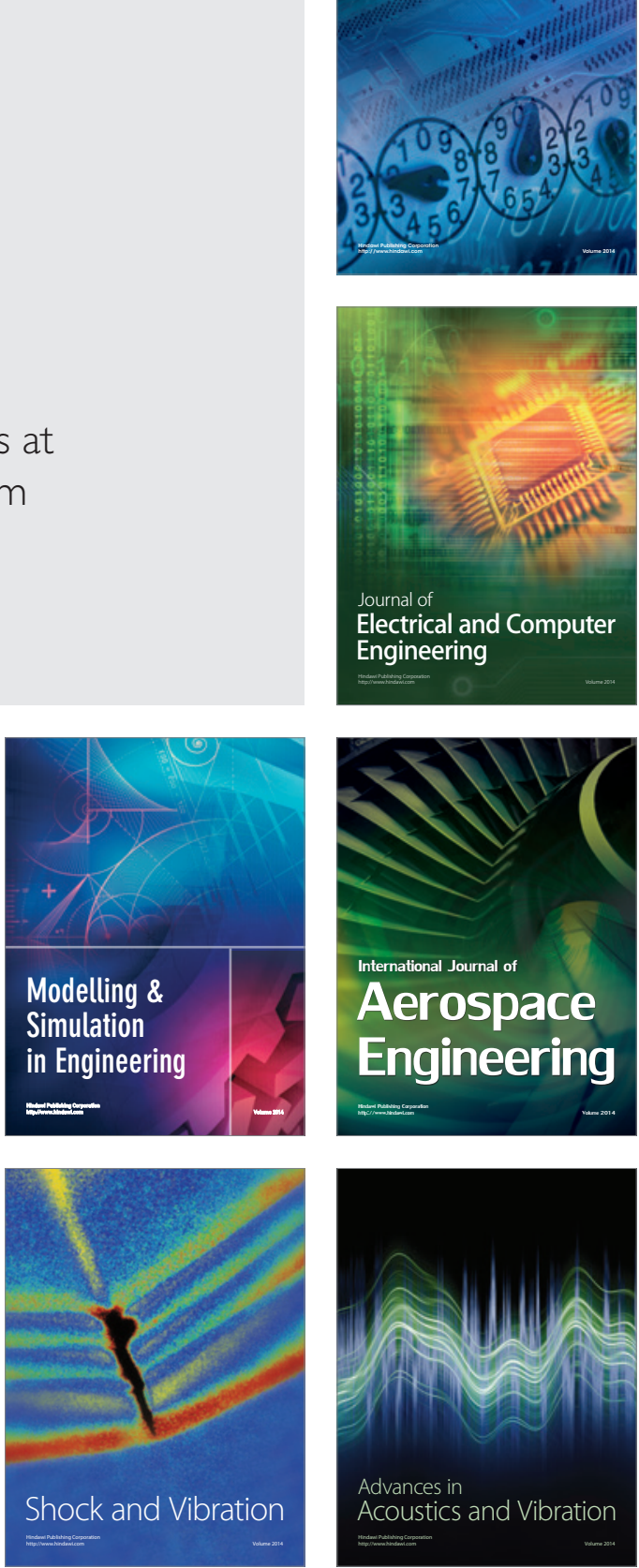